\title{
Article
}

\section{Thermal decomposition of brominated butyl rubber}

\author{
Wei Zhang 3 , Yang Zang ${ }^{3}$, Yanli lư ${ }^{3}$, Weisheng Lin ${ }^{1,2}$, Shengyun Zhao ${ }^{1,2}$ and Jinping Xiong ${ }^{1,2,3 *}$ \\ ${ }^{1}$ School of Ecology and Resource Engineering, Wuyi University, Mount Wuyi, Fujian Provincial \\ 354300, P. R. China; wyuxfyx@126.com \\ ${ }^{2}$ Fujian Provincial Key Laboratory of Ecological Industry Green Technology, Mount Wuyi, \\ Fujian Provincial 354300, P. R. China; wyuxfyx@126.com \\ ${ }^{3}$ School of Materials Science and Engineering, Beijing University of Chemical Technology, \\ Beijing 100029, P. R. China; president@mail.buct.edu.cn \\ *Correspondence: xiongjp@mail.buct.edu.cn; Tel.: +86 1064434908 and +86 1013161551136
}

\begin{abstract}
The thermal decomposition of brominated butyl rubber under air atmosphere was investigated by thermogravimetry (TG) and derivative thermogravimetry (DTG) at various heating rates. The kinetic parameters were evaluated by TG and the isoconversional method developed by Ozawa. One prominent decomposition stage was observed in the DTG curves at high heating rates while an additional small peak was observed at low heating rates. The apparent activation energy determined using the TG method ranged from $219.31-228.13 \mathrm{~kJ} \cdot \mathrm{mol}^{-1}$ at various heating rates. The non-isothermal degradation was found to be a first order reaction, and the activation energy, as determined by the isoconversional method, increased with an increase in mass loss. The kinetic data suggested that brominated butyl rubber had excellent thermal stability. This study will indirectly aid in improving rubber pyrolysis methods and in enhancing the heat resistance of materials.
\end{abstract}

Keywords: Brominated butyl rubber; Thermal decomposition; Lifetime

\section{Introduction}

Brominated butyl rubber (BIIR) is more advantageous than ordinary butyl rubber and is finding increasing applications in many fields [1,2]. Research on the thermal oxidative degradation of BIIR has gained significant attention. This is because BIIR is prone to degradation during long-term use in heat-resistant products, and the study of its performance degradation upon thermal decomposition will aid in developing methods to improve its thermal-aging properties. Additionally, pyrolysis, which is commonly employed in rubber recycling industries, can be effectively utilized to recover resources and energy, thus promoting the progress of waste plastic processing [3]. In this regard, the study on the thermal decomposition kinetics of rubber is an effective approach.

There has been a considerable number of studies on the thermal degradation of rubber materials [4-7] and other polymeric materials [8-10] using different calculation methods and model fitting to experimental data. Liu et al. [4] have studied the effect of vulcanization on the pyrolysis of natural rubber, butadiene rubber, and styrene-butadiene rubber. Based on the activation energy distribution obtained using the distributed activation energy model and the subsequent verification by a model-free method, they suggested that the decomposition of rubber occurred predominantly via a chain reaction. Hu et al. [5] studied the co-pyrolysis of four kinds of real-world plastics and tire in a thermogravimetry-Fourier transform infrared instrument, and kinetic analysis using a single first-order reaction model indicated that the co-pyrolysis occurred in three stages. The DTG curves revealed that the pyrolysis of tire proceeded in two steps, while the pyrolysis of plastics proceeded in a single step. The detailed analysis of the degradation kinetics of EPDM-NBR blend by Basha et al. [6] provided insights into the degradation mechanism. Rasam et al. [7] investigated the kinetics and thermal behavior 
of Spirulina microalgae, sugarcane bagasse, scrap tire and their mixtures from the TG and DTG curves. Based on the kinetic data, they estimated the relationship between the thermodynamic parameters $(\Delta \mathrm{G}, \Delta \mathrm{H}$, and $\Delta \mathrm{S})$ using TG. Das et al. [8] and $\mathrm{Xu}$ et al. [9] studied the thermal decomposition of plastics in the linear heating mode or at a high heating rate by dynamic thermogravimetry. They used model-free methods such as the Ozawa-Flynn-Wall (OFW) method, Kissinger-Akahira-Sunose (KAS) method, and Friedman method for calculating the activation energy. The reaction kinetics were predicted using model fitting methods (including Coats-Redfern and Criado methods). Using isoconversional methods, Das et al. determined the activation energy distribution at each stage of degradation and its influence on the degradation process. Wang et al. [10] investigated the thermal degradation kinetics and non-isothermal crystallization kinetics of mEBHC/HF-PP to predict the thermal stability of HF-PP based on the activation energy obtained using TG analysis and DTG.

The thermal degradation kinetics of BIIR have not been reported yet. In this study, the thermal stability of BIIR was studied by TG and DTG under air atmosphere. TG was performed at different heating rates to study the impact of heating rate on the thermal decomposition of BIIR. To quantitatively study the thermal stability of BIIR, the reaction pathway was obtained from the Crane equation using the kinetic parameters of thermal decomposition, such as the activation energy of decomposition and frequency factor. The kinetic analysis will provide insights into the mechanism of pyrolysis of BIIR, which will subsequently help in improving rubber pyrolysis methods and in enhancing the heat resistance of materials.

\section{Materials and Methods}

\subsection{Raw materials}

BIIR2222 was manufactured by Exxonmobil. The compounding ingredients (phr) were BIIR (100), carbon black N660 (55), sulfur (1), paraffin oil (1), stearate (1.5), zinc oxide (6), and magnesium oxide (2).

\subsection{Specimen preparation}

In the open mill (X (S) K-160 open mill, Shanghai No. 1 Rubber Machinery Factory) at room temperature, BIIR was added, rolled away from $2 \mathrm{~mm}$, thin-film were passed out several times. A vulcameter (P3555C2 vulcameter, Beijing, Central Peak Chemical Machinery experimental works) was used to measure the curing time. Curing was performed for $12 \mathrm{~min}$ in a platen press (25t platen press, Huzhou, Zhejiang Orient Machinery Co., Ltd.) at a curing temperature of $160{ }^{\circ} \mathrm{C}$ to prepare $2 \mathrm{~mm}$ thick specimens of BIIR.

\subsection{TG Analysis}

TG analysis of BIIR in the STARe system thermal gravimetric analyzer (Switzerland METTLER-TOLEDO's product) was performed under air atmosphere at an air flow rate at $20 \mathrm{~mL} / \mathrm{min}$ and heating rates of $0.5^{\circ} \mathrm{C} / \mathrm{min}, 1^{\circ} \mathrm{C} / \mathrm{min}, 2{ }^{\circ} \mathrm{C} / \mathrm{min}, 5{ }^{\circ} \mathrm{C} / \mathrm{min}, 10^{\circ} \mathrm{C} / \mathrm{min}, 20$ ${ }^{\circ} \mathrm{C} / \mathrm{min}$ in the temperature range $30-420^{\circ} \mathrm{C}$.

\section{Result and discussion}

\subsection{Thermal decomposition}

Figure 1 shows the TG-DTG curves obtained at different heating rates. It is evident that there is a rapid weight loss in the temperature range $334.0-389.3^{\circ} \mathrm{C}$ at all the heating rates. This is consistent with the reported melting temperature of BIIR $\left(386^{\circ} \mathrm{C}\right)$ [11]. The weight loss peaks shift to higher temperature with increasing heating rate (Figure 1 and Table 1). Table 1 shows that the decomposition temperature ( $\left.T_{p i}\right)$ of BIIR lies between $334.0{ }^{\circ} \mathrm{C}\left(\right.$ at $\left.0.5{ }^{\circ} \mathrm{C} \cdot \mathrm{min}^{-1}\right)$ and $389.3{ }^{\circ} \mathrm{C}\left(\right.$ at $\left.20^{\circ} \mathrm{C} \cdot \mathrm{min}^{-1}\right)$, while the weight loss is between 
$25.5 \%$ and $28.0 \%$. The molecular chains of the specimen gradually underwent scission with increased heating rate during the thermal analysis. The weight loss peak shifted to a higher temperature as the relaxation time of the molecular chain movement could not keep up with experimental observation time. Because the molecular chain movement and the activation energy of decomposition have an indirect relation with the molecular chain relaxation and temperature, DTG can be employed to analyze the decomposition kinetics of BIIR. A careful inspection of the DTG curves in Figure 1 shows the appearance of an additional peak, corresponding to rapid weight loss, at low heating rates $\left(0.5^{\circ} \mathrm{C} \bullet \mathrm{min}^{-1}, 1\right.$ ${ }^{\circ} \mathrm{C} \bullet \min ^{-1}$, and $\left.2{ }^{\circ} \mathrm{C} \bullet \min ^{-1}\right)$. However, the peak size decreases and the peak ultimately disappears with increasing heating rate. This is because rapid heating promotes pyrolysis at high temperatures, making the reactions to a higher temperature resulting in reaction delayed. However, the reaction steps can be precisely identified at a low heating rate [12].
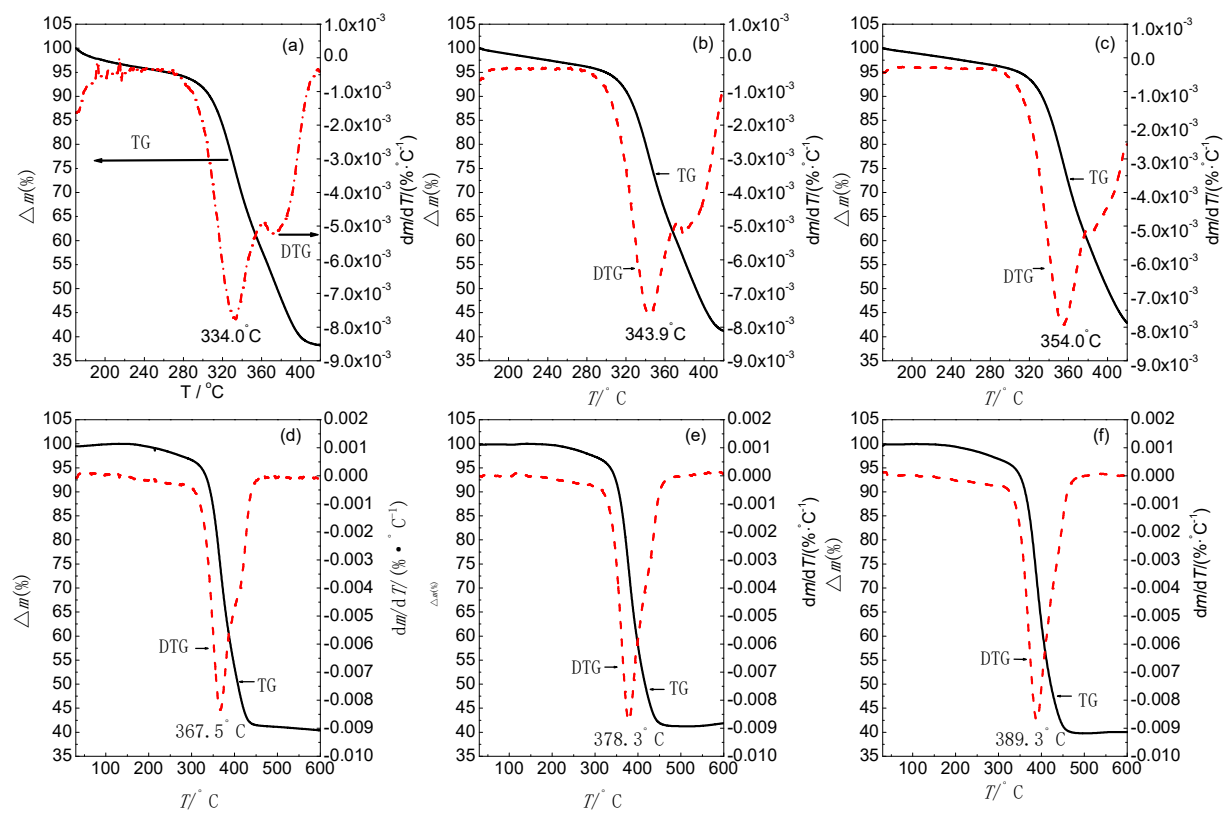

Figure 1. TG and DTG curves of BIIR in air at different heating rates: a) $\left.0.5^{\circ} \mathrm{C} \mathrm{min}^{-1}, \mathrm{~b}\right) 1{ }^{\circ} \mathrm{C} \mathrm{min}^{-1}, \mathrm{c}$ ) $2{ }^{\circ} \mathrm{C} \min ^{-1}$, d) $5{ }^{\circ} \mathrm{C} \mathrm{min}^{-1}$, e) $10{ }^{\circ} \mathrm{C} \mathrm{min}^{-1}$, f) $20^{\circ} \mathrm{C} \mathrm{min}^{-1}$.

Table 1 Decomposition temperatures $\left(\mathrm{T}_{\mathrm{p}} /{ }^{\circ} \mathrm{C}\right)$ of BIIR at different heating rates in air

\begin{tabular}{ccc}
\hline$\beta /\left({ }^{\circ} \mathrm{C} \cdot \mathrm{min}^{-1}\right)$ & $\triangle \mathrm{m}(\%)$ & $\mathrm{T}_{\mathrm{pi}} /{ }^{\circ} \mathrm{C}$ \\
\hline 0.5 & 73.9 & 334.0 \\
1 & 77.2 & 343.9 \\
2 & 76.6 & 354.0 \\
5 & 74.5 & 367.5 \\
10 & 74.3 & 378.3 \\
20 & 72.0 & 389.3 \\
\hline
\end{tabular}

BIIR is prepared by reacting butyl rubber and a small amount of bromine at a certain temperature, and its structure is as follows [13]:

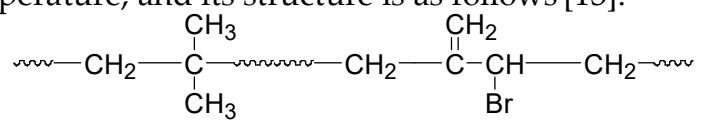


During its thermal decomposition, the -C-Br bond dissociates first owing to low bond energy (Table 2), consequently resulting in the formation of hydrogen bromide at $\sim 230{ }^{\circ} \mathrm{C}$ [14]. However, no significant changes can be observed in the TG/DTG curves (Figure 1) due to the low bromine content in the compound. Rapid weight loss is observed from 330 to $390{ }^{\circ} \mathrm{C}$ primarily due to the dissociation of the $-\mathrm{C}-\mathrm{C}$ - bonds in the main chain. The formation of oligomers upon chain scission is accompanied by the generation of a large amount of hydrocarbon gas in the presence of oxygen, and a sharp weight loss is observed at a lower heating rate in the temperature range $370-380{ }^{\circ} \mathrm{C}$. This weight loss is much smaller than that arising from the dissociation of the main chain [15]. The thermal decomposition pathway can be represented as follows:

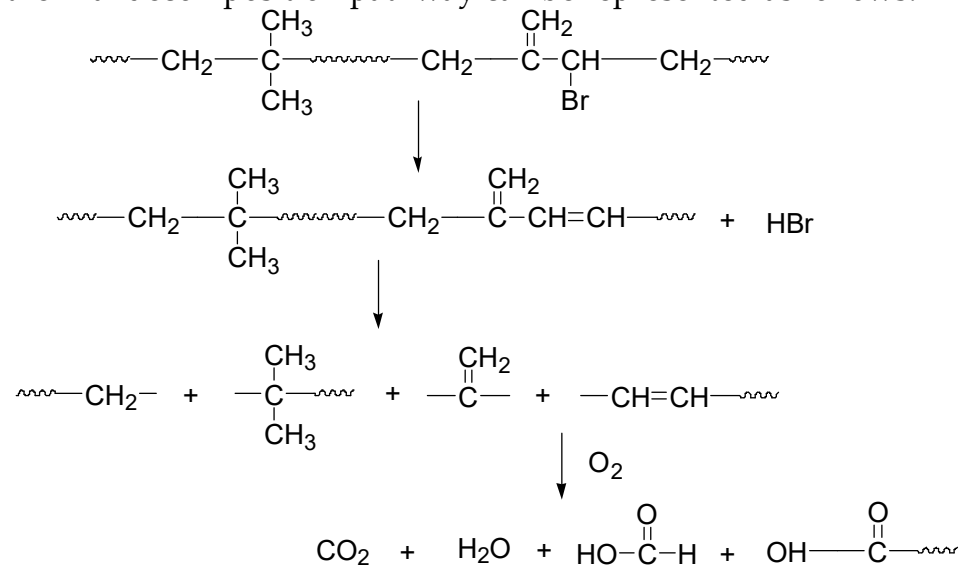

Table 2. Bond energies in BIIR

\begin{tabular}{ccccc}
\hline Bond & $-\mathrm{C}=\mathrm{C}-$ & $-\mathrm{C}-\mathrm{H}$ & $-\mathrm{C}-\mathrm{C}-$ & $-\mathrm{C}-\mathrm{Br}$ \\
\hline $\begin{array}{c}\text { Bond energy } \\
(\mathrm{KJ} / \mathrm{mol})\end{array}$ & 605 & 420 & 346 & 292 \\
\hline
\end{tabular}

\subsection{Activation energy and reaction order of thermal decomposition}

\subsubsection{TG method}

The relation between the extent of weight loss $(\alpha)$ and the time of thermal decomposition can be explained by equation (1) as follows [16]:

$\frac{\mathrm{d} \alpha}{\mathrm{d} t}=A(1-\alpha)^{n} e^{-E / R T}$

Here, A represents the preexponential factor, $\mathrm{E}$ is the activation energy, $\mathrm{T}$ is the absolute temperature, $\mathrm{R}$ is the gas constant, and $\mathrm{n}$ is the reaction order. Under non-isothermal conditions, $\mathrm{A}$ in equation (1) is related to the temperature as $A=A_{0} T^{1 / 2}$. Using this relation, equation (1) can be transformed into equation (2) as follows:

$\frac{\mathrm{d} \alpha}{\mathrm{d} t}=\frac{A_{0}}{\beta} T^{1 / 2} e^{-E / R T}(1-\alpha)^{n}$

Here, $\beta=\mathrm{d} T / \mathrm{d} t$.

An approximated integrated form, equation (3), can be derived after inserting equation (2) for points by Doyle method [17]. The values of E and A can be calculated by the TG method.

$$
\lg \left[\lg \left(1-a^{1 / 2}\right)^{-1}\right]=\lg \frac{A E}{2.3 R \beta}-2.315-0.457 \frac{E}{R T}
$$

An Ozawa plot of $\lg \beta$ against $1 / T$ for different values of $\alpha$ yields a set of parallel straight lines (Figure 2). The values of $E$ and $A$ (Table 3 ) can be obtained respectively from the slopes and intercepts of the straight lines by linear regression, with regression coefficient $r$. The average correlation coefficient $r$ obtained by the TG method is greater 
than 0.99 (close to 1); accordingly, the activation energy calculated by this method is more accurate [18].

The order of thermal decomposition (n) of BIIR can be obtained from the Crane equation, as shown in equation (4)):

$$
\frac{\mathrm{d} \ln \beta}{\mathrm{d}\left(1 / T_{p i}\right)}=-\frac{E}{n R}-2 T_{p i}
$$

A plot of $\ln \beta$ against $1 / T_{p i}$ yields a straight line. The reaction order $n$ (Table 3 ) can be obtained by substituting $\mathrm{E}$ and $\mathrm{T}_{\mathrm{pi}}$ in equation (4). The calculated values of $\mathrm{n}$ are close to 1 , suggesting that the thermal decomposition of BIIR is a first order reaction.

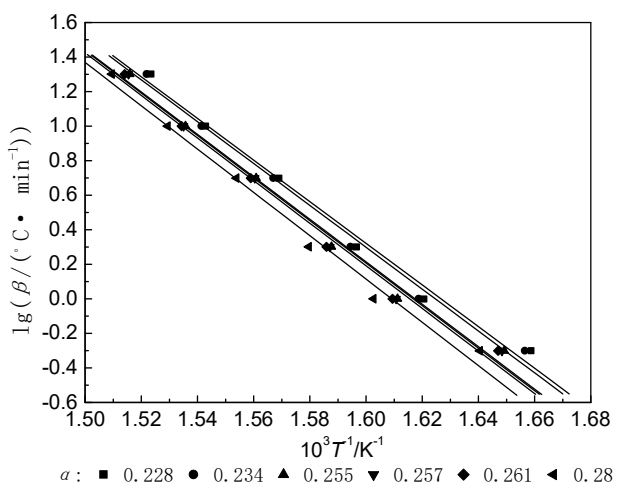

Figure 2. Ozawa plots (based on the TG method) for the activation energy of decomposition of BIIR at different $\alpha$ values.

Table 3 Kinetic parameters of BIIR decomposition

\begin{tabular}{ccccc}
\hline \multirow{2}{*}{$\beta /\left({ }^{\circ} \mathrm{C} \cdot \mathrm{min}^{-1}\right)$} & \multicolumn{4}{c}{ TG method } \\
\cline { 2 - 4 } & $E /\left(\mathrm{kJ} \cdot \mathrm{mol}^{-1}\right)$ & $R$ & $\ln \left(A / \mathrm{min}^{-1}\right)$ & $n$ \\
\hline 0.5 & 224.41 & -0.9946 & 40.67 & 1.05 \\
1 & 219.31 & -0.9949 & 39.87 & 1.03 \\
2 & 220.55 & -0.9946 & 40.08 & 1.04 \\
5 & 223.64 & -0.9943 & 40.56 & 1.05 \\
10 & 224.01 & -0.9945 & 40.62 & 1.05 \\
20 & 228.13 & -0.9940 & 41.29 & 1.07 \\
\hline
\end{tabular}

\subsubsection{Isoconversional procedure (integral method)}

To determine the activation energy $\mathrm{E}$ of BIIR decomposition more accurately, an isoconversional method developed by Ozawa ${ }^{[19]}$ was used. The values of $E$ and $A$ were obtained using equation (5) as follows:

$$
\lg \beta=\lg \frac{A E}{-R \ln (1-\alpha)}-2.315-0.457 \frac{E}{R T}
$$

It is evident that the values of $E$ and $A$ can be obtained from the slope and intercept of the straight line of $\lg \beta$ against $1 / T$ (Figure 3 ) at a particular value of $(1-\alpha)$ (Table 3 ).

Table 3 shows that the activation energy for the thermal decomposition of BIIR is high and is dependent on $\mathrm{E}, A$, and $\alpha$. With increase in the extent of weight loss, the $\mathrm{E}$ 
values tend to increase gradually (Figure 4). It is thus concluded that the dissociation of main chain, which results in weight loss, requires a higher amount of energy.

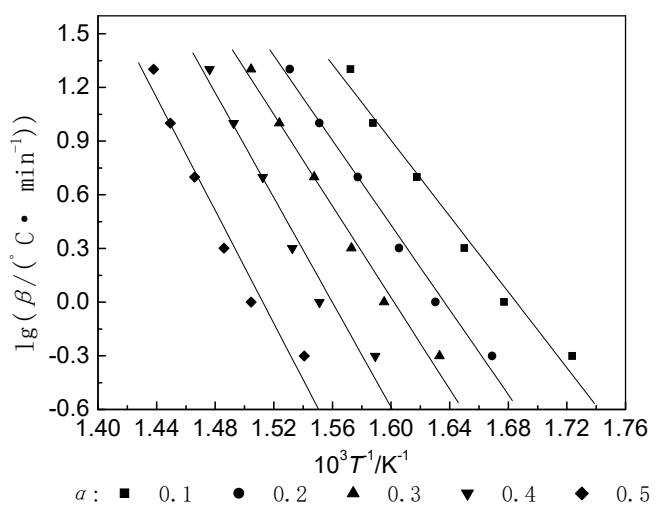

Figure 3 Isoconversional plots of BIIR decomposition in air

Table 4 Apparent activation energy $(E)$ and frequency factor $(A)$ at different fractional mass loss $(\alpha)$ values for BIIR decompostion in air

\begin{tabular}{cccccc}
\hline $1-\alpha$ & 0.9 & 0.8 & 0.7 & 0.6 & 0.5 \\
\hline$E\left(\mathrm{~kJ} \cdot \mathrm{mol}^{-1}\right)$ & 145.32 & 215.33 & 232.66 & 266.50 & 286.75 \\
$\operatorname{Ln}\left(A / \mathrm{min}^{-1}\right)$ & 34.43 & 38.27 & 41.24 & 46.90 & 49.41 \\
\hline
\end{tabular}

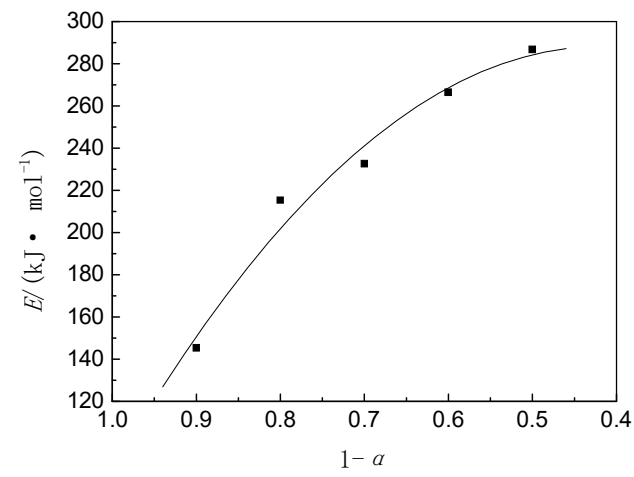

Figure 4 Plot of apparent activation energy (E) for BIIR decomposition in air as a function of $\alpha$

\subsection{Lifetime of thermal decomposition}

The life of polymeric materials is affected by the thermal decomposition during thermal aging even at high temperatures. Thermal aging is also governed by the reaction kinetics, and Equation (6) given by Dakin [20] can be used to predict the lifetime at any temperature.

$$
\lg \tau=\alpha \frac{1}{T}+b
$$

Here, $\tau$ is the lifetime at temperature $\mathrm{T} ; \alpha$ and $b$ are constants derived by integrating Equation (7), which represents the dissociation: 
$-\frac{d \alpha}{d t}=A \alpha^{n} E^{-E / R T}$

Variable separation yields Equation (8):

$\int_{1}^{\alpha}-d \alpha / \alpha^{n}=A e^{-E / R T} \int_{0}^{\tau} d t$

When $\mathrm{n}=1$,

$\lg \tau=E / 2.303 R T+\lg \left[\left(\ln \alpha_{\tau}\right) / A\right]$

$\alpha$ and $\alpha_{\tau}$ are respectively the remaining percentage and the residual percentage at the end of life. Comparing equation (6) and equation (9), we get

$$
\alpha=E / 2.303 R, b=\lg \left(-A^{-1} \ln \alpha_{\tau}\right)
$$

Taking the minimum $\mathrm{E}$ for $219.31 \mathrm{~kJ} \cdot \mathrm{mol}^{-1}$ and the maximum A for $8.55 \times 10^{17} \mathrm{~min}^{-1}$ $(\ln A=41.29)$ in Table 3 , when defined weight loss $5 \%$ to be the index of the end of life for the brominated butyl rubber, it was calculated that $\alpha=1.14 \times 10^{4}, b=-19.22$. Figure 5 shows the life curve, $\lg \tau$ against $1 / T$ of BIIR at $5 \%$ weight loss. The lifetime $\tau$ at a particular temperature and the limit of temperature $T$ at an arbitrary lifetime can be estimated. It is evident that the lifetime could extend to more than 50 years at $30{ }^{\circ} \mathrm{C}$ in air.

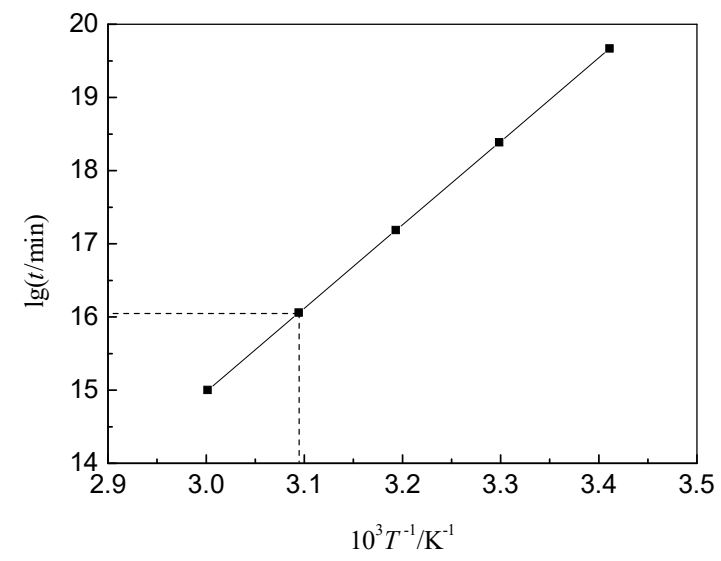

Figure 5 Prediction of estimated lifetime for $\alpha_{\tau}=95 \%$

\section{Conclusions}

The thermal decomposition of BIIR in air proceeds in three steps. The first step is the formation of hydrogen bromide upon the dissociation of the $\mathrm{C}-\mathrm{Br}$ bond; the second step is the dissociation of the main chain; and the third step is the generation of hydrocarbon gases through oxidation. The activation energy of thermal decomposition at various heating rates, as calculated by the TG method, ranged from 219.31 to $228.13 \mathrm{~kJ} \cdot \mathrm{mol}^{-1}$, and the reaction order of thermal decomposition was 1 . Analysis using the isoconversional method developed by Ozawa revealed that the activation energy increased with increase in mass loss. The lifetime estimated from the relationship between the activation energy and weight loss suggested that the lifetime of BIIR was more than 50 years at $30{ }^{\circ} \mathrm{C}$ for a weight loss of $5 \%$. The findings of this study can indirectly help in enhancing the heat resistance of materials.

\section{Patents}


This section is not mandatory but may be added if there are patents resulting from the work reported in this manuscript.

Author Contributions: For research articles with several authors, a short paragraph specifying their individual contributions must be provided. The following statements should be used "Conceptualization, Wei Zhang and Yang Zang.; methodology, Wei Zhang.; software, Liyan Lu.; validation, Wei Zhang, Yang Zang and Weisheng Lin.; formal analysis, Weisheng Lin.; resources, Shengyun Zhao.; data curation, Yanli Lu.; writing-original draft preparation, Wei Zhang.; writing-review and editing, Jinping Xiong.; supervision, Jinping Xiong. All authors have read and agreed to the published version of the manuscript."

Funding: This research received no external funding.

Data Availability Statement: In this section, please provide details regarding where data supporting reported results can be found, including links to publicly archived datasets analyzed or generated during the study. Please refer to suggested Data Availability Statements in section "MDPI Research Data Policies" at https://www.mdpi.com/ethics. You might choose to exclude this statement if the study did not report any data.

Acknowledgments: The authors also would like to thank the Shiyanjia Lab (www.shiyanjia.com) for the FTIR analysis.

Conflicts of Interest: The authors declare that they have no known competing financial interests or personal relationships that could have appeared to influence the work reported in this paper.

\section{References}

1. Cao, R.; Zhao, Xiyuan; Zhao, X.; Wu, X.; Li, X.; Zhang, L. Bromination Modification of Butyl Rubber and Its Structure, Properties, and Application. Ind. Eng. Chem. Res. 2019, 58, 16645-16653. DOI:10.1021/acs.iecr.9b03491.

2. McKeen, L.W. Permeability Properties of Plastics and Elastomer; Vol. 10; Plastic Design Library, 2017.

3. Arabiourrutia, M.; Lopez, G.; Artetxe, Maite; Alvarez, J.; Bilbao, J.; Olazar, M. Waste Tyre Valorization by Catalytic Pyrolysis A Review. Renew. Sustain. Energy Rev. 2020, 129. DOI:10.1016/j.rser.2020.109932.

4. Liu, S.; Jie, Y.; Bikane, K.; Chen, T.; Ma, C.; Wang, B.; Sun, Lushi. Rubber Pyrolysis: Kinetic Modeling and Vulcanization Effects. Energy. 2018, 155.

5. Hu, Q.; Tang, Z.; Yao, Dingding; Yang, H.; Shao, Jingai; Chen, H. Thermal Behavior, Kinetics and Gas Evolution Characteristics for the Co-Pyrolysis of Real-World Plastic and Tyre Wastes. J. Cleaner Prod. 2020, 260. DOI:10.1016/j.jclepro.2020.121102.

6. Basha, SanhaK.T.; Divya, R.; Menon, A.U.; Ashok, N.; Balachandran, M. Cure and Degradation Kinetics of Sulfur Cured Nanocomposites of EPDM-NBR Rubber Blends. Mater. Today Proc. 2018, 5.

7. Rasam, S.; Moshfegh Haghighi, A.; Azizi, K.; Soria-Verdugo, A.; Keshavarz Moraveji, M. Thermal Behavior, Thermodynamics and Kinetics of Co-Pyrolysis of Binary and Ternary Mixtures of Biomass Through Thermogravimetric Analysis. Fuel. 2020, 280. DOI:10.1016/i.fuel.2020.118665.

8. Das, P.; Tiwari, P. Thermal Degradation Kinetics of Plastics and Model Selection. Thermochim. Acta. 2017, 654, 191-202. DOI:10.1016/j.tca.2017.06.001.

9. Xu, Fanfan; Wang, B.; Yang, D.; Hao, J.; Qiao, Y.; Tian, Yuanyu. Thermal Degradation of Typical Plastics Under High Heating Rate Conditions by TG-FTIR: Pyrolysis Behaviors and Kinetic Analysis. Energy Convers. Manag. 2018, 171, $1106-1115$. DOI:10.1016/j.enconman.2018.06.047.

10. Wang, J.; Jia, H.; Tang, Y.; Xiong, X.; Ding, L. Thermal Stability and Non-Isothermal Crystallization Kinetics of Metallocene Poly (Ethylene-Butene-Hexene)/High Fluid Polypropylene Copolymer Blends. Thermochim. Acta. 2017, 647, 55-61. DOI:10.1016/j.tca.2016.11.016.

11. World Rubber Statistics Handbook. Synth. Rubber Industry. 2020, 43, 59.

12. Handbook of Analytical Chemistry, 3rd Edition[J]. Analytical Chemistry. 2018, 46, 1128.

13. Rodgers, B. Rubber Compounding: Chemistry and Applications, 2nd Edition[M].CRC Press:2015-10-09.

14. Handbook of Chemistry and Physics. Anal. Chem. 2016, 44, 1321.

15. Yanbing, W.; Zhixiong, H.; Lianmeng, Z. Study on Vulcanizing and Thermal Behavior of Polymethy/Vinylsiloxane/Butyl Rubber Blend [J]. Adhesion. 2007, 28, 18-20.

16. Lina, Z.; Qi, X.; Zhishen, M.; Xigao, J. Modern Research Methods of Polymer Physics; Wuban University Press: Wuhan, 2003; pp. 233-234.

17. Rosu, D.; Cascavaf, C.N.; Ciobanu, C.; Rosu, L. An Investigation of the Thermal Degradation of Epoxy Maleate of Bisphenol A. J. Anal. Appl. Pyrol. 2004, 72, 191-196. DOI:10.1016/j.jaap.2004.05.002.

18. Sreehitha, P.R.; Durga, B.; Balachandran, M. Dielectric Properties, Thermal Characteristics and Degradation Kinetics of PMMA Nanodielectrics. Mater. Today Proc. 2020, 24. 
19. Run, M.; Dayu, Z.; Sizhu, W.; Gang, W. Studies on Thermal Decomposition of Poly(Ethylene Terephthalate)/Mesoporous Molecular Sieve Composite. Polym. Mater. Sci. Eng. 2006, 22, 64-67.

20. Zhiguo, Z.; Hong, Y. Non-Isothermal Decomposition Kinetics and Lifetime of Polyoxypropylene Ether. J. Zhejiang Univ. Eng. Sci. 2006, 40, 689-692. 